\title{
EL CONFLICTO: UNA ALTERNATIVA DE FORMACIÓN EN LA CLASE DE EDUCACIÓN FÍSICA
}

\author{
CONFLICT AS AN ALTERNATIVE TO TRAINING IN PHYSICAL EDUCATION CLASS
}

Héctor Haney Aguirre-Loaiza ${ }^{1}$
Lady Carolina Vanegas Forero

Resumen

La clase de educación física es un escenario provisto para el encuentro y el desencuentro, donde confluyen acuerdos, disensos y conflictos, que se sitúan en distintas dimensiones del ser humano y convergen en el espacio escolar. El presente artículo aborda aspectos teóricos relacionados con la Educación, que también están asociados a la Psicología Educativa y a la manera como el conflicto entrama diversos factores y subrayando los elementos socio-culturales que se aproximan al fenómeno del conflicto en la clase de Educación Física. De este modo, los conflictos son nutridos y sustentados a partir de los factores socio-culturales y las relaciones que tienen lugar en el mundo cotidiano, como aquellos dados en el entorno familiar, los medios de comunicación, el grupo de pares y los modelos sociales que se visualizan en el desarrollo del aprendizaje social. También se destaca la oportunidad de recalcar el conflicto como abanico expresado en alternativas formativas dentro de la clase de educación física..

Palabras clave: Educación Física, conflicto, psicología educativa, factores sociales, aprendizaje.

\section{Abstract}

The physical education class is a stage provided for the meeting and misunderstanding, a stage where agreements, disagreements and conflicts occur, located on different dimensions of human being and converging in the school environment. This paper focuses on theoretical aspects related to education and associated with educational psychology; also, it approaches the way how conflict interweaves these various factors, and emphasizes socio-cultural elements that involve the phenomenon of conflict in the PE class. This way, conflicts are nurtured and supported from such socio-cultural factors and relationships that occur in the everyday world, such as those provided by family, media, peer groups, and social models displayed in the development of social learning. It also remarks the opportunity to approach conflict as expressed in alternative training range in the physical education class.

Keywords: Physical Education, Conflict, Educational Psychology Social, Learning.

Fecha de recepción: 8 de julio de 2011

Fecha de aprobación: 5 de octubre de 2011

1 Docente del programa de Licenciatura en Educación Física y Deportes de la Universidad del Quindío. Estudiante de Psicología, Universidad de Manizales. Investigador del Grupo Cumanday Actividad Física y Deportes. Correo electrónico: haney34@hotmail.com 2 Estudiante X Semestre - Licenciatura en Educación Física y Deportes, Universidad del Quindío. Correo electrónico: carito2817@gmail.com 
Marco referencial y conceptual del conflicto en el escenario escolar

El conflicto ha constituido elementos fundamentales de la historia social de la humanidad, como también ha estado en presencia de elementos filogenéticos en su evolución natural. No es extraña la presencia del conflicto a lo largo de los pasajes de la historia; ha estado presente en el animismo, la mitología, la religión y hasta en la ciencia. Ésta ha tratado de dar explicaciones desde diferentes disciplinas como la antropología, la biología, la sociología y la psicología.

Tampoco es ajeno a situaciones de la vida cotidiana; se escucha mencionar con bastante frecuencia en expresiones coloquiales sobre los conflictos personales, familiares, emocionales, matrimoniales, laborales, entre otros. Además, se destaca en ello comunicaciones masivas, publicitarias y noticiosas, con la nominación de conflicto para enmarcar un suceso de enfrentamiento social, con matices dados por connotaciones políticas, económicas, territoriales, religiosas y militares. Para Colombia, por ejemplo, se ha abordado la temática del conflicto armado desde hace algunas décadas, de manera que son muchas las facetas en que son expresados y referenciados los diferentes sucesos sociales.

Así mismo, las distintas circunstancias sociopolíticas que enlazan la historia y la actualidad colombiana producen diversos imaginarios negativos respecto a la noción de conflicto, y asociadas a situaciones al vandalismo, violencia, terrorismo, subversión, y cualquier otra cantidad de calificativos que permean el lenguaje escolar. Así, y de acuerdo con lo planteado por Echeverría, Esteve y Jordan en 2001 (citado por Suárez-Basto, 2008) no es apropiado asociar conflicto con violencia. Ambos conceptos se diferencian esencialmente en que, mientras el conflicto responde a situaciones cotidianas, enmarcadas en la vida escolar y mediadas por distintos intereses y debates, por otro lado, la violencia implica enfrentarlas.

Una de las tensiones más profundas concernientes al concepto teórico de violencia, sin desconocer la relevancia de los planteamientos de otros autores sobre el tema, alude a Martín-Báro (2003). Este autor plantea tres enunciados que rigen la violencia, los cuales son configurados para este texto como ejemplos ilustrativos. En primer lugar, están las múltiples formas de la violencia, con un dinámico conjunto de esquemas conductuales; por ejemplo, la violencia legítima ejercida por esquemas de seguridad y defensa de los gobiernos totalitarios y la violencia ejercida por los grupos guerrilleros se diferencia en la legitimidad de las conductas de cada bando. En segundo lugar, Martín Baró plantea el carácter histórico de la violencia entendida desde su mismo contexto social en el que se produce; corresponde a las características particulares y los factores sociales que convergen en el momento especifico en que se presenta el episodio histórico, como la revolución cubana, que en 1959 presentó unas características históricas que afectaron distintos niveles locales (para la cuba misma), continentales (el diseminar un sueño utópico de liberación e igualdad social a través de las armas) e internacionales (en el marco de la Guerra Fría, con la crisis de los misiles en 1962). En tercer lugar, enuncia el Efecto "espiral" de la violencia, uno de los enunciados que más fácil lectura tiene, y que alude a los altos niveles de carga emocional se afloran en comportamientos destinados al rencor, venganza, y odio. Estos ejemplos son dados en un contexto colombiano que emerge de la guerra contra el narcotráfico y se remontan hasta el mismo origen de las FARC (Fuerzas Armadas Revolucionarias de Colombia), con el precedente de la guerra entre los partidos liberales y conservadores agudizado en la década de 1950.

Con el objetivo de hacer una aproximación a los conceptos conflicto-violencia, Pratt (2001) define conflicto como un proceso o situación en la que dos o más seres o grupos humanos tratan activamente de frustrar sus respectivos propósitos e impedir la satisfacción de sus intereses recíprocos, llegando a lesionar o destrozar al adversario; este conflicto puede ser organizado o disuelto, transitorio o permanente, físico, intelectual o hasta espiritual. Por otro lado, y en aras de una definición que abarque las distintas versiones del concepto aceptado de conflicto, seguimos la definición de conflicto que da Paniagua (1980) como la dificultad para conciliar ideas, representaciones, intereses, valores o formas de ver, vivir o entender el mundo.

Es común coligar los conflictos con situaciones negativas o no deseadas, lo que lleva a tratar de evitarlos, evadirlos, ocultarlos e incluso querer eliminarlos, de lo que resulta el rechazo al conflicto. En el énfasis de negar al otro se visualiza la contraposición de ideas que surge ante la necesidad de satisfacción de intereses del individuo y se alimenta de las ansias de eliminar al otro, de quitarlo de escena, y de la competencia entendida desde Maturana (1997), lo cual puede ser contextualizado en lo hasta ahora se desarrolla sobre el tema de conflicto. 
Como ya se ha dicho, la mayoría de las personas, tienden a considerar negar el conflicto como la opción más acertada. Pero el conflicto negado, no enfrentado, conduce a agresividad que deriva muchas veces en violencia. Las amplias concepciones catastróficas y las múltiples asociaciones que se le ha otorgado al conflicto en el plano de conductas no deseadas, ha dificultado la valoración positiva que se puede dar en un contexto de formación como la escuela y en una puesta en escena como en la clase de Educación Física. Pero, ¿quiénes han dificultado este reconocimiento de la negación del conflicto?

Finalmente, una definición que recoge la lógica positiva del conflicto es la señalada por Salinas, Posada, e Isaza, (2002):

Conflicto y ser humano, relación íntima, connatural; contradicción, pugna, lucha, que acerca al hombre a su esencia, que lo potencia como ser capaz de relacionarse consigo mismo, con los otros; que le otorga el beneficio de moverse en estructuras sociales y culturales que le sirven de contenedor, de dique para regular sus relaciones. Espacio que no se puede acabar, ocultar, apaciguar o desconocer, pues es el vector que potencia individuos, grupos, sociedades. Expresión de complejidad, de interconectividad, dinámica de movimiento y transformación, motor de la vida. (p.2)

Desde esta perspectiva es posible visualizar en primer plano un hombre social que no se encuentra aislado en un mundo único o aparte; por el contrario, está en capacidad, de relacionarse con el mundo que le rodea dada esa estructura social en la que se mueve. Por otra parte, se puede vislumbrar un sujeto que en su esencia está predispuesto al conflicto como una condición inseparable de su humanidad, lo cual implica que toda relación social contiene intereses opuestos y elementos de conflicto. La escuela es una organización y como tal su funcionamiento no puede ser entendido sin considerar la signifiาcación del conflicto (Ovejero, 1989).

En esta misma dirección, es importante esclarecer que en el ámbito escolar el conflicto no puede calificarse como una situación de atraso, sino más bien como una situación connatural e inherente al sujeto. Esta visión positiva del fenómeno, lleva a deliberar y reflexionar sobre las posibilidades de avance y progreso que en él se pueden localizar, desde un acercamiento a la esencia del individuo hasta las posibilidades de interacción y relación con el otro y los otros. Dicha realidad no es ajena a la escuela; por el contrario, subyace las prácticas educativas, y en el caso particular, las prácticas de Educación Física. Se conforma así una alternativa de las cuales se pude formar y potenciar el encuentro consigo y los demás. ¿Cómo puede el conflicto ser una alternativa de formación en la clase de Educación Física?

Partiendo de la premisa de que el sujeto es un individuo socializado desde que nace, pues a medida que va creciendo se va insertando y relacionando con el mundo que circunda, se vuelve parte de éste y aprehende sus realidades y dinámicas de funcionamiento. En este contexto, el conflicto que en un inicio pareciera ser un elemento solamente personal, se pone claramente en evidencia en el contexto social; desde esa óptica no sólo se expresa como algo inherente a las sociedades y a las instituciones sino también como un elemento necesario para el cambio social (Jarés,1997).

\section{Sustentadores del conflicto en el ámbito escolar}

Desde una perspectiva general, Viramontes (2000) afirma que son tres los factores que convergen en el espacio escolar: a) los alumnos, b) el docente y c) la institución, rodeada por las políticas que direccionan su funcionamiento. Los estudiantes, lejos de encontrar en las escuelas oportunidades de aprendizaje adecuadas a sus estilos de desarrollo personal, deben aprender a responder a las expectativas y a los estilos de sus profesores y tienen que ingeniárselas para saber cómo combinar sus inquietudes personales de diversas índoles y con sus deberes académicos. Salinas, Posada, e Isaza (2002) afirman en contextos macro, meso y micro sociales que transversalizan al conflicto, con factores como el poco interés de los niños y las niñas por el estudio, el incumplimiento de sus deberes y responsabilidades, la influencia negativa de los ambientes familiares y sociales de donde proceden, las privaciones ocasionadas por el desempleo de sus padres o acudientes y su naturaleza rebelde, que les lleva a desafiar las normas o a reaccionar de manera contestataria. Estas situaciones llevan a demostrar que las situaciones de conflicto no se dan en un solo momento sino que involucran toda una serie de hechos que influyen en la

Respecto al docente, Salinas, Posada, e Isaza (2002) indican que la monotonía de las clases, la poca utilidad de lo que se enseña, la falta de concertación de normas y por ende el desconocimiento de su importancia, que permite que se pase por alto la obligatoriedad de su cumplimiento, invitan al desorden y la desatención e infunden el temor a perder la autoridad frente al grupo 
en los profesores. Estas situaciones generan comportamientos de imposición de autoridad que atribuyen $\mathrm{y}$ asignan mando, pero no respeto, y son condiciones para que un conflicto encuentre una vía de desarrollo, quizás con matices de violencia.

Como tercer factor encontramos a la escuela, donde se pretende una educación estandarizada, donde lo normal se convierte en un principio regulador y todo debe alcanzar los parámetros establecidos por cada institución (Salinas, Posada, e Isaza, 2002). Estos autores también sostienen que en la forma de educación escolarizada actual se traza una media en la cual todos los estudiantes deben alinearse; quien esté por debajo o por encima es excluido de esta dinámica de funcionamiento escolar. Esto se expresa en múltiples formas, que van desde ignorar los hechos hasta el enfrentamiento permanente $y$, en algunos casos, hasta a la expulsión de los estudiantes. Aunado a ello, cabe resaltar que en ocasiones la deficiente infraestructura de algunas instituciones y la escasez de recursos para las prácticas se convierten en factores que afectan de forma negativa el normal desarrollo de las actividades, sobre todo si se tiene en cuenta la naturaleza de la Educación Física. Por lo tanto, éstos pueden ser espacios que contribuyen a agudizar el conflicto y donde se presentan con violencia las difusas líneas fronterizas de determinadas particularidades.

Lo que se desenvuelve en torno a la escuela y las condiciones sociales en las que se desempeña el niño alimenta y sustenta el conflicto y hace más complicado su tratamiento e intervención. Como es un proceso que se da y se fortalece por los factores sociales,las condiciones económicas, el entorno familiar, el entorno social y cultural, los medios de comunicación, entre otros. En el entorno hay un fácil acceso al conflicto y por consiguiente éste permea estos factores en la medida en que la escuela es un contexto donde converge diversidad de pensamientos, ideas e intereses, que tienen soporte en las construcciones sociales dadas desde el escenario familiar, social y cultural y que concurren en el ámbito escolar.

La Educación Física como escenario institucionalizado de aprendizaje por modelado en la clase de Educación Física

El aprendizaje por modelado, también identificado como aprendizaje social, (Bandura y Walter, 1974; Bandura y Ribes, 1978) es un modelo de aprendizaje que explica claramente cómo diversos factores ambientales son determinantes en las conductas del sujeto, y cómo el sujeto afecta el ambiente. Maturana (1997) explica, por ejemplo, que si en los padres es común resolver las discusiones con gritos, empujones o golpes, el niño hará lo mismo en el espacio escolar, de una manera $\mathrm{u}$ otra. A ello se unen otros elementos que aceleran $\mathrm{u}$ obstaculizan dicho proceso, como el contexto en el que vive, el grupo de pares, el núcleo familiar, medios de comunicación, religiones y creencias, etc., que esencialmente son considerados por la psicología social como factores de riesgo o de protección, de acuerdo con los comportamientos entretejidos en el contexto (micro, meso y macro).

Del mismo modo, el docente se convierte en un modelo permanente, lo cual constituye un reto y sobre todo un compromiso que debe reconocer a la multiplicidad de acciones que se entrelazan en la vida de los educandos a cargo, cada uno de los cuales tiene expectativas, sentires, pensares, aconteceres y actuares diferentes entre sí. El sujeto aprehende la dinámica social a medida que crece y se relaciona con ella y se apropia de sus realidades al crear pactos y formas de interactuar con dicha sociedad; comprende cómo es que ésta funciona y las formas de relacionarse para poder mantenerse en ella, como un ser activo y coherente con su funcionamiento. Entonces, es necesario reconocer que todo ese bagaje del individuo constituye información fundamental en sus esquemas mentales y procesos psicológicos (atención, sensación, percepción, memoria, lenguaje, pensamiento, aprendizaje, etc.) que en tal relación social convergen; por lo tanto, se verá afectado en su comportamiento, así como en su dimensión espiritual, su cuerpo y su dimensión afectiva-emocional.

Así, un escenario escolarizado (para este caso, la clase de Educación Física) se convierte en un espacio donde convergen múltiples factores medio-ambientales y el docente es un modelo para el joven; las interacciones entre los estudiantes y el profesor son determinadas por el clima de aula (Ascorra, Arias, y Graff, 2003). Además la propia dinámica de la clase de Educación Física brinda la posibilidad de salir del puesto delimitado por un pupitre, encontrarse con el otro e interactuar con toda la realidad que a ambos circunda y determina su comportamiento ante diferentes situaciones que se presenten en la clase. Según lo planteado, ese espacio es propicio para encuentros y desencuentros que marcan diferencias respecto a otros espacios académicos que ocurren la mayoría de las veces dentro del aula de clase y que en ocasiones desconoce la esencia del estudiante 
como ser social, y quizás al desconocer tal esencia se desconocer la naturaleza misma del ser humano y por consiguiente del conflicto. Si bien en la escuela, como se comentó anteriormente, se ven matices de conflicto, en la clase de educación física se dan más por la esencia misma de vincular a los niños en el patio.

De esta manera, el conflicto se convierte en una oportunidad de formación que tiene precedentes en las interacciones sociales que propias del área de Educación Física, que aquí son mayores que en las demás áreas (Sánchez-Arroyo, et al, 2008). Estos espacios están diseñados por elementos mediadores que no sólo permiten correr o hacer giros, pases y lanzamientos técnicamente limpios, sino que le brindan la posibilidad de desarrollar habilidades comunicativas, autonomía, pensamiento crítico, capacidades de argumentación y proposición ante las diferentes situaciones cotidianas. Estas prácticas proporcionan una formación en competencias básicas, que le permiten al sujeto responder a las exigencias de la sociedad en la que se ve inmerso, deben hacer de la clase de Educación Física el escenario eje de transformación del reconocimiento de su cuerpo y de la relación que puede crear a partir de él con el otro y los otros.

Este tipo de práctica de la Educación Física encuentra una vía de desarrollo en la medida en que se asuma como una disciplina pedagógica que se preocupa por el sujeto en formación, a partir del conflicto y en el ejercicio de significar al ser humano como ser social. Esto da la posibilidad de ver cómo las construcciones sociales que se imbrican en el sujeto, y las cuales constituyen un sujeto y una realidad que se hacen, se piensan, se permean en él, se sienten y viven en su realidad. Así, el conflicto puede ser la resultante de todo un conglomerado de situaciones y factores sociales que lo soportan y que, ante el descuido, pueden convertirse en factores de riesgo. Sin embargo, con la convicción del maestro educador físico y el papel transformador de la educación, dichos factores de riesgo pueden convertirse en factores de protección, con la consiga que el conflicto es la oportunidad de educar desde la potencialidad y no desde la carencia.

Como conclusión Jarés (1993) señala que ser docentes con la capacidad de educar en el conflicto es posible en la medida en que se parta de una actitud positiva y sensible ante los estudiantes. Se debe confrontar lo que se estudia en el aula con la experiencia de la vida; tratar de comprender, profundizar y tomar una posición precisa ante los conflictos; cuestionar el conformismo, la complicidad, la obediencia y la pasividad que educa para la desobediencia. Cascón (2000) afirma que "para educar en el conflicto, habrá que buscar espacios en los que profesorado y alumnado se preparen y desarrollen herramientas que les permitan abordar y resolver los conflictos con mayor creatividad y satisfacción". También formula que es necesario tener en cuenta aspectos que nos permitirán acercarnos de alguna forma a la posibilidad de educar en el conflicto: crear grupos en un clima de afecto y confianza, puesto que para él "todas las personas tenemos dos necesidades humanas muy básicas: el sentimiento de pertenencia a un grupo y el de identidad" (Cascón, 2000 s.p.). Así mismo, este autor exhorta a crear espacios donde se favorezca la comunicación, con el diálogo como un aliado muy funcional y pertinente, a aplicar la toma de decisiones por consenso, fomentando la participación, y trabajar la cooperación, ya que esto permite conocer las percepciones de todas las partes y encaminarnos hacia el reconocimiento del otro.

Entonces, no es posible pensar en que el docente de Educación Física se quede inmóvil, desprevenido ante el conflicto. En la medida que quiera hacer uso de herramientas para potenciar a sus estudiantes, es preciso que se informe, actualice sus conocimientos, adopte como bandera la educación desde la potencialización y no desde la carencia, para estar en capacidad de responder a las necesidades propias de una sociedad que cambia velozmente y exige profesionales, no sólo de titulo, sino también de vocación y compromiso con su labor formadora, y que luego del accionar en el patio, reflexionen sobre sus prácticas y a partir de ello sean capaces de formular nuevas estrategias que propendan por una mejor acción educativa. Esto debe reflejarse en los estudiantes que están formando, no sólo desde las prácticas en las que no hay discusiones y todo está aparentemente en paz, sino también desde situaciones en las que intervengan en las tensiones de los escolares y el reconocimiento y tratamiento oportuno de los conflictos se conviertan en una posibilidad de formación en los encuentros de Educación Física.

Ascorra, P., Arias, H. y Graff, C. (2003) La escuela como contexto de contención social y afectiva. Revista enfoques educacionales 5 (1): 01 - 15, 2003

Bandura, A. \& Ribes, E. (1978) Modificación de la Conducta. México DF., México: Trillas.

Bandura, A. \& Walter, R.H. (1974) Aprendizaje Social y Desarrollo de la Personalidad. Madrid, España: Alianza. 
Cascón, P. (2000) Educar en y para el conflicto Cátedra UNESCO sobre Paz y Derechos Humanos. Barcelona, España. Recuperado el 27 de noviembre de 2010 en http://escolapau.uab.cat/img/programas/educacion/publicacion005e.pdf

Jarés, X. (1993) El lugar del conflicto en la organización escolar. Revista Iberoamericana de Educación. Volumen (15). Recuperado el 23 de noviembre de 2010 en http://www.rieoei.org/oeivirt/rie15a02.htm

Martín-Baró, I. (2003) Poder, ideología y violencia. Madrid: Trotta

Maturana, H. (1997) Emociones y Lenguaje en Educación y Política. (9aed.) Santiago de Chile: Dolmen.

Paniagua M. (1980) Los signos del conflicto y su resolución. Revista Educar. Jalisco México. Recuperado el 11 de diciembre de 2010 en http://www.google. com.co/url?sa=t\&source=web \&cd=3\&ved=0CCMQ FjAC\&url=http://preparatorialomas.org/uploads / allimg/29Educar\%20Conflictos\%20Escolares. pdf\&rct $=j \& q=$

Ovejero, A. (1999) Psicología social de la educación. Barcelona: Herde

Pratt, H. (2001). Diccionario de Sociología. México.DF: Fondo de Cultura Económica.

Salinas, M.L., Posada, D.M. \& Isaza, L.S. (2002) A propósito del conflicto escolar. Revista Educación y Pedagogía. Volumen XIV, No. 34. Recuperado el 15 de febrero de 2011 en http://revinut.udea.edu.co/index.php/ revistaeyp/article/viewFile/5934/5344

Salinas, M.L., Posada, D.M. \& Isaza, L.S. (2002) A propósito del conflicto escolar. Revista Electrónica interuniversitaria de formación del profesorado 5(4). Recuperado el 29 de noviembre de 2010 en http://web.archive.org/web/20041220211000/www.aufop.org/ publica/reifp/articulo.asp?pid=211\&docid=873.

Sánchez-Arroyo, J.F., Chinchilla, J.L., Carmona, M., Romero-Ramos, O. (2008) Las relaciones sociales y educativas existentes entre los elementos personales del proceso educativo durante una sesión de Educación Física. Un estudio de casos. Retos. Nuevas tendencias en Educación Física, Deporte y Recreación. 14, 66-69. Suárez-Basto, O.E. (2008) La mediación y la visión positiva del conflicto en el aula, marco para una pedagogía de la convivencia. Revista Diversitas-Perspectivas en Psicología. 4(1), 187-199

Viramontes, G. (1980) Educar en el conflicto: Un elemento dinamizador de las prácticas educativas. Revista Educar. Jalisco México. Recuperado el 24 de enero de 2011 en http://kino.iteso.mx/ gerardpv/ doctorado/educarenelconflicto.html 